\title{
Les Second Fruits de John Florio ou la vie comme un jeu
}

\author{
Alessandro Arcangeli \\ Traducteur : Nicole Abi Aad Spitaleri
}

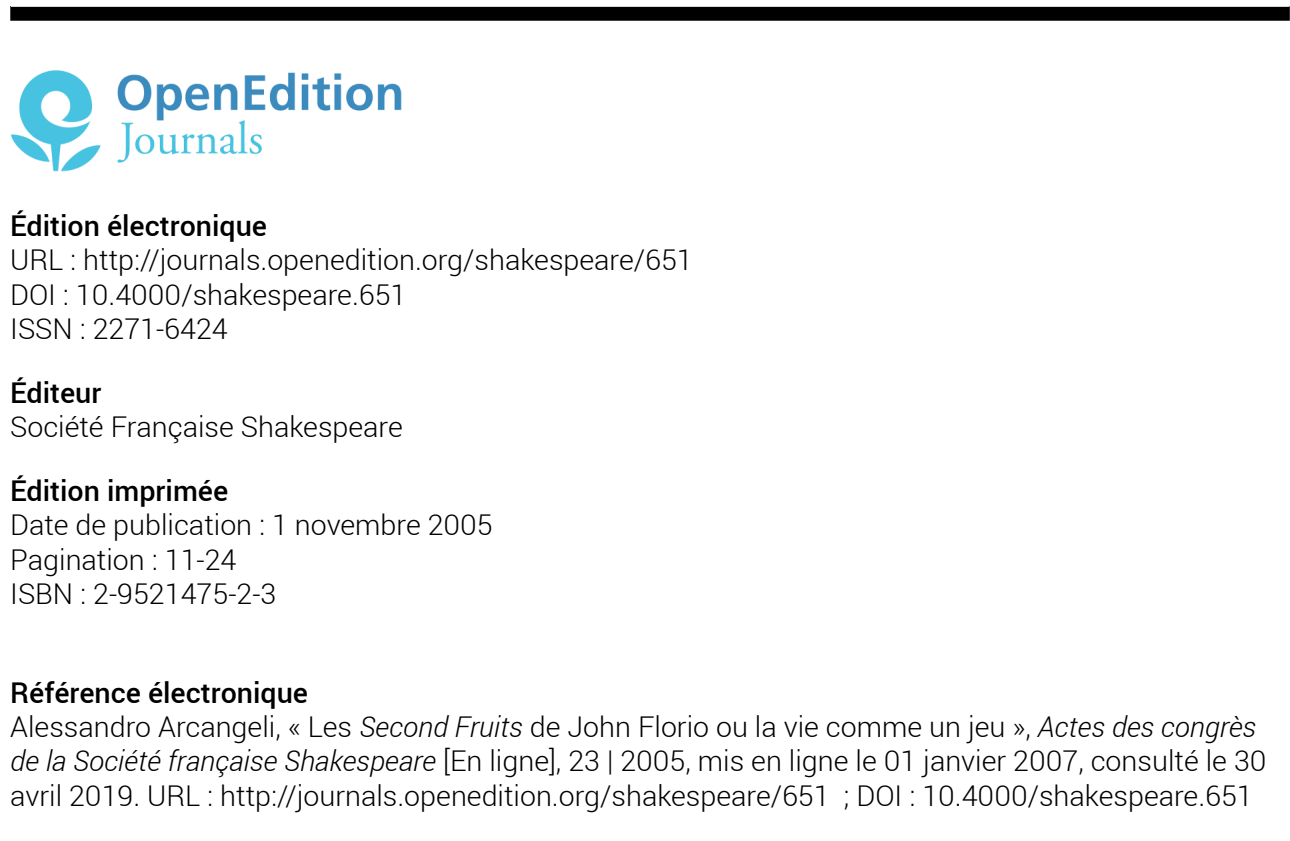




\section{Shakespeare et le jeu}

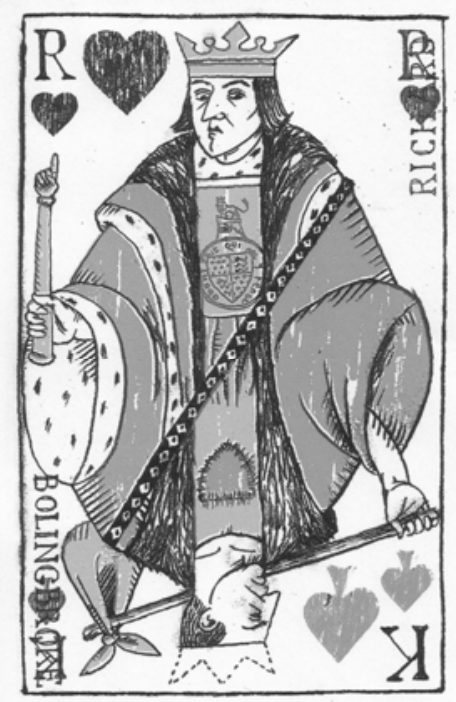

actes du Congrès organisé par la

SOCIÉTÉ FRANÇAISE SHAKESPEARE les 10, 11 et 12 mars 2005

textes réunis par Pierre KAPITANIAK sous la direction de Yves PEYRÉ 


\title{
COMITÉ SCIENTIFIQUE :
}

\author{
Margaret Jones-Davies \\ Gisèle Venet \\ Jean-Marie Maguin \\ Yves Peyré \\ François Laroque \\ Pierre Kapitaniak
}

COUVERTURE :

Edouard Lekston, King and Kusin, 2005

edlek@free.fr

conception graphique et logo

Pierre Kapitaniak

(C) 2005 Société Française Shakespeare

Institut du Monde Anglophone

Université de Paris III - Sorbonne Nouvelle

http://recherche.univ-montp3.fr/SFS/

5 rue de l'École de Médecine

75006 Paris

ISBN 2-9521475-2-3

Tous droits de traduction, de reproduction et d'adaptation réservés pour tous les pays 


\title{
LES SECOND FRUITS DE JOHN FLORIO OU LA VIE COMME UN JEU
}

\begin{abstract}
Alessandro ARCANGELI
II est notoire que, parmi les manuels de conversation italienne pour les lecteurs anglais à l'époque élisabéthaine, les Second Fruits (1591) de John Florio marquent, par rapport aux précédents First Fruits (1578), un passage des tons moralisants de l'éthique protestante à ceux plus détendus de la conversation agréable. La critique a noté, depuis longtemps un rapprochement entre le texte de Florio et les manuels italiens de bonnes manières, comme étant le lieu d'élaboration de l'art de la conversation propre à la Renaissance. Si cela constitue, déjà en lui-même, vu les formes du discours dialogique, un espace ludique, les jeux et les loisirs fournissent par la suite explicitement un objet privilégié des conversations elles-mêmes, qui deviennent donc métaludiques. De plus, la discussion sur le jeu alterne avec la description des jeux effectivement joués par les interlocuteurs. Des sports et des passe-temps pratiqués ou objets de discussion (du jeu de paume à l'équitation, des cartes au trictrac et aux échecs, sans oublier le voyage, l'amour ou les facéties) on peut reconstituer la journée d'un gentilhomme modèle - évidemment un modèle de langue, mais aussi de comportement. Le "Jardin de récréation ", la liste des proverbes qui accompagnent l'édition, mais qui sont également utilisés dans le texte des dialogues, sera lui aussi examiné comme faisant partie intégrante de l'opération culturelle proposée par l'auteur.
\end{abstract}

It is well known that, among Italian conversation manuals for English Elizabethan readers, John Florio's Second Fruits (1591) marked, compared to his previous First Fruits (1578), a passage from the moralizing tone of Protestant ethics to the more relaxed one of polite conversation. $A$ connection between Florio's text and Italian treatises on manners - the birthplace of the Renaissance art of conversation - is a long-established critical acquisition. Given the fact that the text is a dialogue, its very genre assigns it to the world of a literary play. Moreover, games and pastimes are an explicit and favourite topic of the conversations, which therefore become a form of play on play. Furthermore, within the text, discussion on play alternates with the description of games actually played by the interlocutors. From the sports and pastimes which we read of as either performed or discussed by Florio's personae (from real tennis to horse riding, from cards to backgammon and chess, not to forget travel, love or jokes) we can reconstruct the average day of a typical gentleman. This was clearly a set conversation, but also a model of behaviour. The "Garden of Recreation" - the list of proverbs that follow in the printed edition but which are also used within the text of the dialogues - will also be considered as an integral part of the author's cultural project.

Q uand on explore la composante ludique de la culture élisabéthaine, il faut tenir compte des manuels de conversation en langue étrangère pour le public anglais, avec une attention particulière pour les manuels italiens. À cette époque, on assista à la plus grande floraison du genre, car dans la deuxième moitié du $\mathrm{XVI}^{\mathrm{e}}$ siècle, le prestige de la langue et de la culture italienne en Angleterre atteignirent leur apogée. Les résistances et les hostilités ne manquèrent 
pas, et un "inglese italianato ${ }^{1}$ » était proverbialement quelqu'un à éviter. Reste le fait qu'un ensemble de facteurs se combinait pour faire jouer à certains textes, thèmes et personnages de provenance italienne un rôle qu'ils n'avaient pas auparavant et qu'ils perdirent par la suite. Comme on le sait bien, en plus d'une influence générale de la culture de la Renaissance, un rôle important fut joué par les réfugiés protestants - aussi bien italiens que français - qui avaient fui en Angleterre, et qui avaient trouvé leur place en tant que médiateurs culturels et linguistiques, en enseignant leur propre langue maternelle et en traduisant les livres étrangers en anglais. L'affiliation religieuse de ces professionnels des échanges culturels du $\mathrm{XvI}^{\mathrm{e}}$ siècle ne manqua pas d'influencer leurs œuvres et leurs textes. Quand le réfugié florentin Michelangelo Florio, professeur d'italien de Lady Jane Grey, doit donner dans sa grammaire un exemple d'utilisation du conditionnel, il propose l'exemple suivant: «Si j'obéissais au pape, à l'antéchrist j'obéirais ${ }^{2}$ ». Par la suite, Florio mourut en Suisse, où il s'était réfugié après l'expulsion décrétée par Mary Tudor, mais une fois qu'Élisabeth accéda au trône, les protestants du Continent eurent la possibilité de rentrer en Angleterre et de reprendre leur activité. Un des plus en vue parmi eux est le français Claude de Saliens (alias Holyband), qui nous fournit un autre exemple de propagande religieuse par le biais de l'enseignement linguistique: s'étant réfugié outre-Manche après le déclenchement de la guerre de religion dans sa mère patrie, il prit avec lui un traité de sévère censure calviniste contre la danse. Ce traité avait

\footnotetext{
${ }^{1}$ John Florio, Second Frutes, 1591, éd. R. C. Simonini Jr, Gainesville, FL, Scholars' Facsimiles \& Reprints, 1953 (et coll. The English Experience, Amsterdam-New York, Theatrum Orbis Terrarum-Da Capo Press, 1969), « To the reader » : «Un inglese italianato, è un diavolo incarnato ». Voir Frances Amelia Yates, John Florio : The Life of an Italian in Shakespeare's England, Cambridge, C.U.P., 1934, chap. 1 ; Ead., "John Florio's Father » (1955) et «Italian Teachers in Elizabethan England » (1937-8) dans ses Collected Essays, vol. 2, Renaissance and Reform, Londres, Routledge-The Warburg Institute, 1983, p. 16180 ; Spartaco Gamberini, Lo studio dell'italiano in In ghilterra nel '500 e nel '600, MessineFlorence, D’Anna, 1970. Après la rédaction du présente article Michael Wyatt a fait paraitre une contribution majeure à l'étude de Florio traducteur et lexicographe: The Italian Encounter with Tudor England: A Cultural Politics of Translation, Cambridge, C.U.P., 2005 .

${ }^{2}$ Giovanna Perini, «Florio, Michelangelo » dans Dizionario biografico degli italiani, vol. 48, Rome, Istituto della Enciclopedia Italiana, 1997, p. 379-81; Owe Boersma, « Florio, Michael Angelo » dans Oxford Dictionary of National Biography, éd. H. C. G. Matthew et Brian Harrison, Oxford, O.U.P., 2004, vol. 20, p. 168-9.
} 
été publié semi clandestinement en France en 1564, et il le réimprima, après une révision stylistique, en appendice à son fameux manuel de langue française pour Anglais, comme un exercice de lecture et de prononciation. L'opération semble avoir eu quelque succès, du moins à en juger par le fait que le traité contre la danse fit l'objet de traductions anglaises, aussi bien manuscrites qu'imprimées ${ }^{3} \ldots$ Quelques années après de Saliens, le fils de Michelangelo, John Florio, était occupé dans une entreprise semblable, faisant pratiquement la même chose avec l'italien. Ses First Fruits (1578) contiennent en effet une condamnation tranchante du théâtre et une tirade moralisante contre la futilité de l'amour, inspirée par le prédicateur impérial Antonio de Guevara ${ }^{4}$.

Le ton est différent cependant quand, après treize ans, ses Second Fruits (1591) paraissent. Il y a un passage bien connu où on discute de théâtre d'une manière critique, mais en démontrant une certaine familiarité avec la scène contemporaine, et en abandonnant les condamnations sans appel 5 . Quant à l'amour et aux femmes, il en discute dans un chapitre attitré (le dernier). Mais en général l'atmosphère a changé, elle s'est détendue pour virer vers la conversation agréable. Le motif pour lequel on s'y intéresse ici est, plus spécifiquement, le fait que jeux et divertissements occupent un espace exorbitant à l'intérieur du texte. Pour pouvoir apprécier les

\footnotetext{
3 Alessandro Arcangeli, Davide o Salom è? Il dibattito europeo sulla danza nella prim a età moderna, Trévise-Rome, Fondazione Benetton Studi Ricerche-Viella, 2000, p. 140-1, 1489 .

4 John Florio, His Firste Fruites, London 1578, coll. The English Experience, AmsterdamNew York, Theatrum Orbis Terrarum-Da Capo Press, 1969. Voir Clara Longworth de Chambrun, Giovanni Florio : un apôtre de la Renaissance en Angleterre à l'époque de Shakespeare, Paris, Payot, 1921 ; Vincenzo Spampanato, «Florio, John» (1923) dans son Sulla soglia del Secento : studi su Bruno, Campanella ed altri, Milan, Società editrice Dante Alighieri, 1926, p. 69-126 ; Frances Amelia Yates, John Florio, op. cit. ; Desmond O'Connor, «Florio, John » dans Oxford Dictionary of National Biography, op. cit., p. 1658. Quant à Carlo Maria Franzero, John Florio a Londra ai tem pi di Shakespeare, Parme, Guanda, 1969, il s'agit d'un plagiat éhonté du livre de Yates, comme l'a déjà justement remarqué Giovanna Perini, "Dialogo didattico e dialogo drammatico: John Florio e William Shakespeare », Studi secenteschi, ${ }^{\circ} 33,1992$, p. 167-82.

5 John Florio, Second Frutes, op. cit., p. 23 :

«GIOvanNI. And then after dinner we will goe see a plaie.

HENRICO. The plaies that they plaie in England, are not right comedies.

THOMASO. Yet they doo nothing else but plaie every daye.

HENRICO. Yea but they are neither right comedies, nor right tragedies.

GiovanNI. How wou you name them then?

HENRICO. Representations of histories, without any decorum. »
} 
implications de ce choix nous devons partir du contexte, de la tradition au sein desquels le livre se place.

Les manuels de Florio n'ont certainement pas inventé un genre littéraire, et se limitent à réinterpréter, en la renouvelant, une habitude qui avait déjà donné lieu à une pluralité d'expériences significatives, avec lesquelles il vaudra la peine d'établir quelques points de contact. Dans le domaine de la didactique des langues vulgaires, l'Angleterre de la fin du XIve siècle et du début du Xve siècle avait produit une série de manières de langage, petits manuels en forme de dialogue qui enseignaient le français parlé (anglo-normand tardif) en recourant à la simple méthode de l'apprentissage mnémonique des phrases. Les situations prévues comprennent - comme dans les guides de voyage de nos jours - formules de salutation, demander l'heure et le chemin, et dialogues à l'auberge. Les chapitres de la Manière de 1396, en outre concernaient le corps humain, l'aménagement d'une maison, le départ en voyage, en plus d'une variété de contextes professionnels. À son tour la Manière de 1399 contenait le lexique de l'année ecclésiastique, les numéraux, monnaies, animaux, injures et insultes, et plus spécifiques " manières pour parler aus dames et aus damoiselles ${ }^{6}$ ». Ensuite, il existait le filon bien exploité des dialogues latins, le prototype de la didactique de la langue, à qui l'humanisme avait donné un nouvel élan. Il n'y manquait pas la représentation de scènes ludiques. Un chef d'œuvre de ce genre, que sont les Colloques d'Érasme, contenait un chapitre dédié aux jeux de balle, au lancer du poids et au saut, parmi d'autres qui traitaient d'ultérieures activités de loisir, comme la chasse. Même l'Exercitatio linguae Latinae du collègue espagnol d'Érasme, Juan Luis Vives, eut une large circulation européenne et aussi anglaise, et a été indiqué comme la source originelle de la méthode didactique que Florio fit sienne. Cette dernière suit la journée d'un écolier entre la maison et l'école (Florio l'imite également en adoptant comme première scène le fait de se lever le matin), et contient deux dialogues dédiés au jeu de cartes et aux règles du jeu.

\footnotetext{
${ }^{6}$ Manières de langage (1396, 1399, 1415), éd. Andres M. Kristol, Londres, Anglo-Norman Text Society, 1995.
} 
En premier lieu, il faut dire que dans une culture, comme celle de la Renaissance italienne et européenne, où la conversation ellemême était considérée comme la forme fondamentale du jeu ou du divertissement, le premier élément ludique qu'il est impossible de ne pas remarquer dans le texte de Florio est sa propre nature dialogique. La conversation est traitée expressément comme un passe-temps quand, dans le titre de deux chapitres, il nous est dit qu' « on raisonne sur beaucoup de choses agréables » («many pleasaunt discourses ») et le thème même du fait de converser peut être le « parler familier tard le soir » («familiar speech late in the evening7 »). La composition des dialogues qui régit les douze chapitres n'est pas toujours la même. On part du cas, le plus fréquent, de l'échange entre les interlocuteurs de phrases courtes qui s'étendent sur une ligne, à l'entraînement à tenir un discours plus ample et articulé. On trouve deux chapitres qui sont plus longs que les autres, le sixième qui est dédié aux voyages, et le dernier, qui, comme on l'a dit, traite de l'amour et des femmes ; mais leurs dimensions sont liées essentiellement au fait qu'ils fonctionnent comme réservoirs de nombreux proverbes (parfois même peu liés au thème dominant, mais insérés là, profitant de l'occasion offerte par la conversation). Ce sera l'occasion alors de rappeler que l'édition des Second Fruits est accompagnée du Giardino di ricreazione, un ensemble de plus de six mille proverbes italiens, mis par ordre alphabétique et non traduits, précédés de ses propres frontispices et paratextes - un document des plus précieux en son genre. À l'intérieur des dialogues qui précèdent, l'auteur a tendance à utiliser le plus grand nombre possible de proverbes du Giardino, en en offrant ainsi des exemples de contextes possibles tout comme, dans ce cas, une traduction. À tel point qu'on a relevé le fait que les Second Fruits sont un instrument au service du Giardino di ricreazione, plutôt que l'inverse. L'image du jardin («dans lequel poussent des feuillages, des fleurs et des fruits vagues, gracieux et suaves $\left.{ }^{8} »\right)$ et sa fonction déclarée

\footnotetext{
7 John Florio, Second Frutes, op. cit., chap. 4 et 10.

8 John Florio, Giardino di ricreatione, frontispice, publié à la suite de son Second Frutes, op. cit.
} 
récréative augmentent sans doute le poids de l'élément ludique au sein de la publication.

Le langage du loisir s'impose dès le début du premier colloque. Nolano et Torquato sont les interlocuteurs. Nolano représente non seulement Giordano Bruno, l'ami de Florio, qui avait été en Angleterre de 1583 à 1585, mais aussi plus spécifiquement le personnage autoréférentiel que Bruno lui-même place dans un de ses dialogues, une promenade à travers les lieux londoniens de la mémoire. Il rend visite à Torquato le matin dans sa chambre, et le trouve encore au lit. Le personnage de Bruno se préoccupe immédiatement de ne pas brusquer son ami : ils ont le temps, il n'y a aucune hâte.

Levatevi a vostro bell'agio : ch'io v'aspetterò bene [...]. Non tanta Rise at your leisure : for I will stay for fretta : nò. you [...]. There is no such hast ${ }^{9}$.

La question de la hâte est reprise plus loin, parce que Nolano est surpris de trouver l'autre encore au lit. Il le souligne avec une exclamation, et il s'engage dans une discussion sur l'heure la plus opportune pour se lever, et s'il est déshonorant de rester au lit trop longtemps. Les deux concluent avec une morale sur le ton de la plaisanterie :

O se la poltroneria fosse virtu : $\quad$ Oh if sluggishnes were a vertue, quanti virtuosi sarebber'al mondo. how many vertuous men would there $\mathrm{be}^{1 \mathrm{O}}$ ?

Puis le gentleman paresseux harcèle son domestique pour qu'il lui passe ses vêtements (et en passant il le menace et jure presque- ce sont toutes des occasions linguistiquement très formatives pour enseigner au lecteur un lexique utile). Nolano lit en attendant (c'est une première activité spécifique de loisir: la lecture de salle d'attente), avant de reprocher de nouveau à son ami sa lenteur. Ici Torquato peut citer l'un des premiers proverbes de l'ouvrage :

Chi va piano va sano.

Soft fier makes sweet malte ${ }^{11}$.

9 John Florio, Second Frutes, op. cit., p. 2-3.

${ }^{10}$ Ibid. 
Quelquefois Bruno s'énerve et mitraille lui-même une bonne ration de proverbes, avant de bénir Dieu parce que son ami est finalement prêt. Mais avant de sortir, c'est le moment du petit déjeuner (les repas : un autre lieu commun des manuels pour apprendre une langue étrangère).

Le petit déjeuner est à base de pain et de vin. Si Nolano profite de ce moment pour déclarer ses habitudes austères, le sujet est un de ceux qui, tout au long de l'œuvre, permettent aux interlocuteurs de s'occuper de médecine, avec des termes où la frontière entre culture populaire et culture savante n'est pas trop nette. À cette époque, on voyait des gens communs s'intéresser fréquemment au sujet de la santé, sans laisser ce monopole aux médecins. La médecine populaire avait un véhicule fondamental de transmission grâce aux proverbes, et le fait que l'œuvre de Florio soit constellée de proverbes, aide certainement à mettre en relief cette thématique. Par exemple, dans un des dialogues suivants, on voit un serviteur qui s'était blessé au bras en tombant dans les escaliers. Le patron, soutenant qu'il voulait le soigner, lui tire le bras dans un premier temps, puis lui prescrit de le garder lié à la poitrine toute la journée. Profitant de la visite à une tierce personne, les deux interlocuteurs cités auparavant parlent ensuite du fait qu'il a de la fièvre, et des saignées qu'il a faites pour se soigner ${ }^{12}$.

Le deuxième chapitre entre dans le vif de l'activité ludique en mettant en scène un jeu de paume, une situation discursive qui avait déjà été esquissée par de Sainliens, et que Florio développe en y ajoutant une série de détails, qui ont déjà attiré l'attention des historiens du tennis. On y trouve inclue, par exemple, la référence à la pratique de déposer la mise du jeu sous la corde (qui remplaçait notre filet moderne).

Parmi les interlocuteurs du troisième dialogue, l'un rend visite à l'autre, puis décide d'aller à cheval et à la chasse. Le choix de Florio de représenter le fait que l'un d'eux possède deux chevaux (un pour lui, et un autre pour ses amis), alors que l'autre possède deux oiseaux de proie et trois genres de chiens de chasse est un bon indicateur du rang

\footnotetext{
${ }^{11}$ John Florio, Second Frutes, p. 10-11.

12 Ibid., p. 42-47.
} 
social des personnages. Naturellement, ces listes servent essentiellement à enseigner au lecteur le plus grand nombre possible de mots italiens ; mais toutes les situations montrent des personnes qui essayent toujours avec peine de trouver des occupations pour remplir leur temps : ainsi, une fois décidé ce qu'ils allaient faire, ils louent un cheval pour deux ou trois jours, sans qu'ils semblent retenus par d'autres engagements. Dans le quatrième dialogue, on assiste à un déjeuner somptueux, où un certain convive mange à tel point qu'il se sent mal - de nouveau, on a la richesse du vocabulaire au centre, mais avec des ouvertures intéressantes sur les habitudes et les mentalités : si la nourriture et le vin abondent, l'eau est considérée juste bonne pour se laver les mains. Tout de suite après avoir mangé, on demande aux serviteurs d'apporter les cartes; et le cinquième chapitre est dédié spécifiquement au jeu. Le choix de l'activité est expressément fait pour «tromper le temps » (« to deceave the time $\left.{ }^{13} »\right)$. On parle d'aller faire une promenade, mais le temps atmosphérique le déconseille; il convient donc de « jouer à quelque jeu » (« plaie at some game $\left.{ }^{14} »\right)$. On évite bien d'aller dans une maison de jeu ( « at some common dycing house $\left.{ }^{15} »\right)$, parce qu'il s'agit delieux mal famés qui contrastent fortement avec le statut social et la conduite morale des personnages de Florio. Le choix des jeux, précise-t-on, est lié au temps (atmosphérique) et à la compagnie, mais cela va des jeux de balles (soit le tennis, soit le ballone) et des boules aux jeux de table. Parmi les jeux de cartes, on choisit la primera, et on y joue avec un jeu de cartes françaises (avec «des trèfles, des piques, des carreaux et des cœurs $\left.^{16} »\right)$. En jouant les amis conversent, et quelques conversations ont pour sujet la chance.

S'ensuivent des parties de trictrac et d'échecs ; de ces derniers, on s'attarde également à décrire l'origine et la ressemblance avec l'art militaire. $\mathrm{Vu}$ qu'il fait encore mauvais (après tout, nous sommes

\footnotetext{
13 John Florio, Second Frutes, p. 65.

14 Ibid.

15 Ibid., p. 67.

${ }^{16}$ Ibid., p. 69.
} 
toujours à Londres), on renonce une seconde fois à sortir et la compagnie se déplace pour lire dans le bureau de l'hôte.

Le septième dialogue s'ouvre également sur un échange de plaisanteries sur ce qu'on peut faire, entre des interlocuteurs qui ont recours à un proverbe efficace pour éclaircir leur rapport avec toute activité professionnelle :

Et io faccio il mestier di Michelazzo, mangio, bevo, e vado a solazzo.
And I am free of Michaels occupation, I eate and drinke, and walke for recreation ${ }^{17}$.

Une fois sortis de la maison, ils n'ont pas de destination précise : l'un suggère d'aller où le hasard les porte, l'autre propose l'école d'escrime. Cela offre l'occasion de nommer - ne fût-ce qu'avec les initiales Vincenzo Raviolo, le maître italien qui était récemment arrivé en Angleterre, et qui en 1595, publiera le premier traité anglais d'escrime. Un des deux interlocuteurs prend des leçons avec ce maître et, revenant encore une fois sur la question de l'emploi du temps, « il ne sait que faire d'autre ${ }^{18}$ " (qu'apprendre à faire de l'escrime). En plus d'être escrimeur, Saviolo est un danseur expert, et permet ainsi à Florio de nommer des mots clés de la sociabilité du xvI ${ }^{\mathrm{e}}$ siècle comme la pavane et la bravoure. Le texte cependant ne nous accompagne pas jusqu'à l'école, car chemin faisant les deux amis se souviennent qu'ils veulent acheter quelque chose, et la conversation se détourne pour présenter le lexique du shopping (en dédiant un large espace à la négociation sur le prix d'un achat).

Des femmes, dans l'œuvre, on parle inévitablement en plusieurs occasions, pour en dire autant de bien que de mal ; entre autre, en reprenant, au huitième chapitre, le topos des parties qu'une belle femme doit avoir. Le matériel du dernier chapitre est organisé en regroupant les proverbes et les observations pour ou contre les femmes en deux blocs opposés, et en leur attribuant deux interlocuteurs distincts. L'effet est, ainsi, celui d'une controverse sur la noblesse relative des deux genres, ce qu'on pouvait lire dans d'autres œuvres

\footnotetext{
${ }^{17}$ John Florio, Second Frutes, p. 114-15.

18 Ibid., p. 116-17.
} 
littéraires de l'époque, et en particulier dans le chef-d'œuvre du genre, le troisième livre du Cortegiano. L'intervention la plus longue, qui s'étend sur deux pages, est celle du défenseur des femmes, Silvestro, qui utilise comme argument apologétique le fait qu'une grande partie des meilleures choses ou de celles qui sont les plus importantes du monde - des forces de la nature aux concepts abstraits les plus importants - sont, en italien, grammaticalement féminins (un raisonnement qui fonctionne beaucoup moins dans la traduction anglaise, où tous ces termes sont linguistiquement neutres) ${ }^{19}$. Comme la critique l'a déjà depuis longtemps reconnu, c'est l'une des étapes qui révèle un modèle littéraire que Florio avait encore plus directement présent, c'est-à-dire l'ami Giordano Bruno (il s'inspire, spécifiquement, des dialogues de De la causa, principio, et uno, où il assumait la fonction de louange de la reine Élisabeth).

En parlant des Second Fruits, d'autres ont déjà signalé leur rapprochement avec l'art de rédaction des traités qui, au XVI ${ }^{\mathrm{e}}$ siècle en Italie, définissait et divulguait les canons de l'art de la conversation. Le rapport de Florio avec cette expérience culturelle est complexe. D’un côté, il est clair et inévitable qu'un manuel de conversation doive contenir des situations et des formules qui font partie d'un répertoire plus général des manuels de bonnes manières. Au bout du compte, il s'agit toujours aussi d'apprendre à éviter de faire piètre figure dans une série de contextes. En outre, il semble que l'auteur a bien l'intention de profiter de l'occasion pour enseigner à son public quelques manières plus raffinées, que les modèles culturels italiens sont en mesure de proposer aux élites des autres pays. Tout comme Bruno, Florio ne mâche pas ses mots pour critiquer ça et là ce qu'il considère être de mauvaises habitudes des Anglais (à partir des excès à table) ${ }^{20}$.

Cependant, John Florio, qui ne manquait pas de se référer de façon critique dans son œuvre à des adversaires et à des propositions culturelles alternatives en en prenant ouvertement ses distances, s'en prend - à un certain point de la dédicace à Nicholas Saunder - à ces

\footnotetext{
${ }^{19}$ Ibid., p. 198-205.

${ }^{20}$ Gian Napoleone Giordano-Orsini, "L'Inghilterra di Shakespeare descritta da G. Florio », Civiltà moderna, 1932, p. 70-85.
} 
« strangers, who they think hath learnt a little Italian out of Castilion's courtier, or Guazzo his dialogues ${ }^{21} »$. La référence est, dans ce passage, spécifiquement linguistique, parce que Florio est en train de faire de la publicité à son dédicataire pour son recueil de proverbes, et accuse ceux qui croient avoir appris l'italien de cette façon, d'être livresque («bookish ») ${ }^{22}$. Même si Florio lui-même était né à Londres et n’a probablement jamais mis les pieds en Italie, il déclare que ses proverbes viennent de là-bas - en effet il en tire un bon nombre des œuvres de l'ami Bruno, et de celles d'autres auteurs contemporains. Proverbes mis à part, il a été observé que les First Fruits empruntent du matériel d'un auteur linguistiquement vivant et peu orthodoxe comme Anton Francesco Doni; et que, même si Florio rend les passages en question plus pédants que ce qu'ils n'étaient à l'origine, le fait même qu'il choisisse un tel modèle est le signe d'une certaine liberté des canons stylistiques dominants ${ }^{23}$. On relève la même ampleur et variété des textes de référence dans ce qui est considéré comme le chef d'œuvre de Florio, c'est-à-dire son dictionnaire italienanglais. Pourtant, en retournant à la pointe lancée contre les lecteurs étrangers de Castiglione et de Guazzo, la langue n'est probablement pas tout. Malgré les points de contact répétés avec le monde du Cortegiano, la sociabilité qui occupe les interlocuteurs des dialogues de Florio est urbaine, et pas courtoise : elle serait inconcevable hors de son contexte londonien. Ainsi, après un échange de phrases infinies de courtoisie entre les interlocuteurs d'un dialogue - l'un a accepté de la part de l'autre le prêt d'un livre et un cadeau qui consiste en un tableau - il en vient à dire :

Di gratia lasciamo queste ceremonie da canto, convenienti piu tosto a finti odierni cortegiani, che alla nostra candida \& sincera amicitia.
I pray you let us leave these ceremonies a parte, as more beseeming our counterfaite courtiers this daye, than our pure and unspotted friendship.

\footnotetext{
${ }^{21}$ John Florio, Second Frutes, op. cit., The Epistle Dedicatory.

22 Ibid.

23 D. G. Rees, « John Florio and Anton Francesco Doni », Comparative Literature, 1963, p. $33-8$.
} 
Et l'autre convient d'être « très contraire aux cérémonies » qui « entre les amis ne devraient pas se faire ${ }^{24} \ldots$ » Le topos pluriséculaire du mépris de la cour, revitalisé au XvI ${ }^{\mathrm{e}}$ siècle par Guevara, est repris d'une manière plus diffuse dans le neuvième chapitre, où on discute expressément des courtisans. On arrive ainsi dans l'ensemble à un compromis anglo-italien intéressant, qui regarde vers l'avant, aux formes des relations sociales des siècles successifs, plutôt qu'aux standards précédents de l'étiquette. Et toutefois, celle qui est représentée par Florio relève toujours de la sociabilité aristocratique et non bourgeoise, comme l'attestent le statut social des personnages et certaines des activités qui les occupent.

Les occupations en question sont presque toujours des activités de loisir. Un rare exemple d'affaires traitées par un des interlocuteurs est représenté, à un certain moment, par l'occupation d'un d'entre eux à la Bourse de Londres, qui pourtant se résout en un instant lors d'une conversation avec une autre personne avec qui il a rendez-vous - et il s'agit d'une conversation hors scène, qu'on ne propose pas au lecteur du dialogue comme exercice linguistique. Tout compte fait, les modèles culturels et linguistiques proposés se déroulent toujours et en tout cas dans la vie sociale de l'élite, qui par définition doit affronter chaque activité de la journée comme un passe-temps. À l'arrière-plan du tableau, il y a les serviteurs, souvent maltraités parce qu'ils sont lents ou distraits dans l'accomplissement de leurs propres devoirs (à un certain point, un serviteur a la malheureuse idée de demander qu'on lui énumère ses défauts, et il se voit présenter une longue liste qui de nouveau est un collage de proverbes ${ }^{25}$ ). Un observateur extérieur aurait du mal à distinguer qui des personnages est le plus oisif. Le fait que la paresse reste le délit principal dont on accuse ses propres subordonnés de la part d'un groupe social qui se vante justement de ne pas bien savoir comment passer la journée est l'indice le plus clair possible qui montre combien la gestion du temps est étroitement liée au statut social et aux rapports de pouvoir.

\footnotetext{
24 John Florio, Second Frutes, op. cit., p. 34-35.

25 Ibid., p. 134-35.
} 
Il y a une autre caractéristique fondamentale qui sépare la convivialité représentée par Florio de la convivialité représentée par Castiglione et de son milieu de référence : si on parle de femmes, les femmes sont pourtant tout à fait absentes de la conversation - un autre choix qui rapproche ces dialogues du modèle proposé par Giordano Bruno.

Vers la fin du XvI e siècle, des dialogues didactiques comme ceux de Florio sortirent de la scène de la littérature anglaise, tandis que les dialogues dramatiques des écrivains de théâtre y faisaient leur entrée. Entre les deux expériences, cependant, il y a eu des échanges significatifs dans les deux sens, et également à propos des Second Fruits il a été noté qu'ils évoquent par-ci par-là une partie du style de la comédie de l'époque ${ }^{26}$. Si des suggestions plus ou moins récentes, disant que Florio a été le vrai auteur du théâtre de Shakespeare, peuvent prêter à sourire ${ }^{27}$, et même si la fréquentation personnelle entre les deux peut être raisonnablement imaginée mais n'est pas explicitement documentée, et même si l'identification de Florio comme étant la personne dont s'inspirent certains des personnages shakespeariens n'a pas convaincu grand monde, il reste attesté que Shakespeare utilisa de façon récurrente des passages tirés des écrits de Florio. Au-delà des emprunts spécifiques, l'intermédiaire culturel le plus influent entre l'Italie et l'Angleterre de la fin du XVI siècle a laissé des traces dans le contexte de la littérature anglaise contemporaine qui l'a imité même quand elle voulait le critiquer $^{28}$ - ainsi qu'au sein des puissants cercles aristocratiques qu'il fréquentait plus directement. On dirait plutôt que c'était un intermédiaire pas seulement avec l'Italie, si on pense à son rôle comme traducteur des Essais; d'ailleurs ce rôle était au centre du colloque Shakespeare et Montaigne ${ }^{29}$. En lui, a trouvé ainsi un intermédiaire efficace, également, cette culture ludique

\footnotetext{
${ }^{26}$ Giovanna Perini, « Dialogo didattico e dialogo drammatico », op. cit.

${ }^{27}$ Santi Paladino, Shakespeare sarebbe il pseudonimo di un poeta italiano, Reggio-deCalabre, Borgia, 1929. Mais voir aussi les références à des hypothèses plus récentes dans Desmond O'Connor, « Florio, John », op. cit., p. 168.

28 Pour le cas de John Eliot, voir Frances Amelia Yates, John Florio, op. cit., p. 139-73.

${ }^{29}$ Shakespeare et Montaigne : vers un nouvel humanisme, éd. Pierre Kapitaniak et JeanMarie Maguin, Paris, Société Française Shakespeare, 2004 (voir particulièrement les communications par Warren Boutcher et Philippe Desan).
} 
spécifique qui avait été élaborée par certaines générations dans les cours et dans les villes de l'Italie de la Renaissance. Il est impensable que Shakespeare n'en ait pas été influencé quand il caractérisait des personnages pris dans des activités de loisir.

Alessandro ARCANGELI
Università di Verona

(traduction de Nicole Abi Aad Spitaleri) 